\title{
Using the Method of Medical Insurance Payment Reform to Promote the Path Selection of Large Hospital Development
}

\author{
Pengyu Xu \\ School of Business Administration, Andrews University, Berrien Springs, USA
}

\section{Email address:}

pengyu@andrews.edu

\section{To cite this article:}

Pengyu Xu. Using the Method of Medical Insurance Payment Reform to Promote the Path Selection of Large Hospital Development. Science Journal of Public Health. Vol. 7, No. 2, 2019, pp. 53-58. doi: 10.11648/j.sjph.20190702.14

Received: April 19, 2019; Accepted: May 20, 2019; Published: May 23, 2019

\begin{abstract}
At the beginning of the establishment of the basic medical insurance system in China, the primary implementation was a single project-based payment. At present, it has gradually explored a single or mixed way to pay, mainly including payment by disease type, payment by the number of people, payment by medical service unit and payment by the DRGs (Diagnosis Related Group System). This paper discusses the reform of payment mode of basic medical insurance in large hospitals and summarizes the main features of the reform of the payment mode of basic medical insurance in the past. First of all, the basic medical insurance management department has absolute choice and pricing power. The government is responsible for the management of basic medical insurance, and the hospital is in a state of passive implementation of government policies. In addition, the reasonable demands of large hospitals can hardly be effectively reflected in the policy. Last but not least, the improvement of hospital serviceability and the promotion of patients' reasonable medical treatment effect have not been realized effectively. The research results of this paper mainly discuss the reform path of basic medical insurance payment methods in large hospitals. For one thing, large hospitals can proactively report to the basic medical insurance management department the reformed disease types and disease charges for basic medical insurance payments. For another thing, the large hospitals can determine the diagnosis and treatment plan of the disease according to the clinical path of disease diagnosis and treatment. The basic medical insurance management department can explore the new path method of reforming the basic medical insurance payment method in large hospitals according to the reform idea of "the same disease insurance standards are consistent" and individual "differentiated self-payment." At the same time, it is recommended that the government use the professional management ability of commercial insurance companies to improve the performance of basic medical insurance by purchasing commercial insurance company services. Give play to the third-party role of commercial insurance companies to help alleviate the asymmetry of the hospital and patient medical information and the contradiction between doctors and patients.
\end{abstract}

Keywords: Large Hospitals, Reform of Medical Insurance Payment Methods, Differentiated Self-Payment, The Same Disease Insurance Standards Are Consistent, Basic Medical Insurance Management Department, Reform of Medical and Health System

\section{Introduction}

In March 2009, the Central Committee of the Communist Party of China and the State Council on deepening the reform of the medical and health system, proposed to build a four-in-one basic medical and health system. [1] It is mainly formed by covering public health service systems, medical service systems, medical security systems, and drug supply security systems for urban and rural residents. The four major systems complement each other, supporting construction and coordinated development. Among them, the medical service system, medical security system, and drug supply security system involve medical care, medical insurance, and medicine. The effective linkage between them constitutes the important reform of China's current medical and health system reform. At present, the main contradiction in the field of medical and health care in China is essentially the contradiction between the rapidly growing demand for medical services by the people and the development of medical and health services. [2] There are mainly contradictions that do not match or mismatch. The emergence of these contradictions is mainly due to the unreasonable 
supply mechanism of medical services. In fact, because of the difficulty of seeing a doctor (Due to the unequal allocation of medical and health resources, urban and rural residents will often go to the municipal, provincial or national large-scale medical institutions for diagnosis and treatment after suffering from a serious illness. Then, the current Chinese grading diagnosis and treatment system has not been established effectively, and the problem of disorderly medical treatment for seriously ill patients has not been solved effectively. Therefore, the number of patients treated in these large medical institutions has formed a problem of difficulty in seeing a doctor.) and the existence of expensive medical treatment (After suffering from major illnesses, urban and rural residents usually go to municipal, provincial or national large-scale medical institutions for treatment. However, the cost of diagnosis and treatment of these large medical institutions is relatively high, and the level of basic medical insurance for urban and rural residents is low. Therefore, the level of protection for basic medical insurance for urban and rural residents in large hospitals is also at a low level, and residents generally need to bear higher medical expenses.), there is a contradiction between mismatched or mismatched between the rapidly growing medical service demand of urban and rural residents and the development of medical and health service supply. Based on this situation, the essence of deepening the reform of the medical and health system is the "supply-side reform" in the field of health care. [3] The reform of payment methods for basic medical insurance (including basic medical insurance for urban workers and basic medical insurance for urban and rural residents) is one of the important contents for deepening the reform of the medical and health system. It is to promote the behavioral norms of hospital medical services, the external driving force and endogenous motivation of hospitals according to their own development needs. [4] Through the reform of basic medical insurance payment methods, large-scale hospitals can be purposefully upgraded (Generally refers to a three-level large general hospital, a three-level large specialized hospital, a government-level national or provincial or municipal large hospital.) Self-management enthusiasm, guide the scientific allocation of medical services resources in large hospitals and promote the development of large-scale hospitals from the scale expansion to the special disease diagnosis and treatment of key specialist diseases. From the perspective of the above-mentioned national policies, the scientific and rational selection of the reform path of basic medical insurance payment methods plays a significant role in promoting the development of large hospitals and improving the self-management of large hospitals. [5] Promoting the reform of basic medical insurance payment methods is an important task for China to deepen the reform of medical and health systems and the management of basic medical insurance. At the same time, it is also an important measure to effectively control the unreasonable growth of medical expenses, ensure the safety of basic medical insurance funds, and alleviate the contradiction between hospitals and patients.

\section{Main Features of the Reform of China's Current Basic Medical Insurance Payment Methods}

In June 2017, the Guiding Opinions of the General Office of the State Council on Further Deepening the Reform of the Payment Mode of Basic Medical Insurance clearly put forward that the budget management of the Basic Medical Insurance Fund should be further strengthened, and the reform of the payment mode of basic medical insurance should be carried out in an all-around way, which is mainly based on the payment of diseases and combined in various ways. [6] In each region, a certain number of diseases should be selected to implement payment by disease type. The country will select some regions to carry out pilot projects based on the payment by the DRGs (Diagnosis Related Group System). The state encourages all localities to improve payment methods such as payment by the number of people, payment by the medical service unit. By 2020, the reform of basic medical insurance payment methods will cover all medical institutions and medical services. Multi-composite basic medical insurance payment methods that are universally implemented and adapted to different diseases and different service characteristics nationwide. In the end, the proportion of payment by the project is significantly reduced. [7] At present, a lot of exploration and practice have been carried out in the process of reforming the payment method of basic medical insurance in various parts of China, and some results have been achieved. From the practice of various places, the reform of basic medical insurance payment methods mainly reflects the following common features.

\subsection{The Basic Medical Insurance Management Department Has Absolute Choice and Pricing Power}

The basic medical insurance management department has given a uniform, and clear regulations in the form of documents in the important aspects of disease selection, diagnosis and treatment route selection, price standards, payment standards, etc., as the hospital are basically in a "passive" implementation state in the reform. Although the basic medical insurance management department can consider the differences between different levels of hospitals in the unified disease charging standards and payment standards, the differences between hospitals of the same level, especially large hospitals, are often difficult to reflect.

\subsection{The Reasonable Appeal of Large Hospitals Is Difficult to Be Reflected Effectively in the Policy}

There is no effective interaction mechanism between the basic medical insurance management department or the specific business handling department and the large hospital. The negotiated pricing mechanism based on the reform of basic medical insurance payment methods cannot be established effectively. It is easy to cause controversy in the professional aspects of the diagnosis and treatment of 
diseases such as the clinical path of disease types. From the perspective of the main objectives of the basic medical insurance reform, the basic medical insurance management department or the specific business handling department is mainly aimed at controlling the unreasonable increase in the costs associated with the diagnosis and treatment of diseases. They often pay insufficient attention to the quality of medical services for diagnosis and treatment of diseases and the effectiveness of disease diagnosis and treatment. This often leads to a low willingness and enthusiasm for large hospitals to implement basic health insurance payment reforms.

\subsection{Failure to Effectively Improve Service Capabilities and Promote Reasonable Medical Treatment}

The reform of basic medical insurance payment methods has played a role in controlling diseases that do not meet the diagnosis and treatment, and unreasonable medical expenses. However, basic medical insurance is based on the budget amount under total control. Under normal circumstances, the growth rate of medical service demand cannot be kept up. At the same time, new diagnostic and therapeutic technologies, as well as new drugs, continue to emerge. This situation often results in a large difference between the total budgetary expenses of basic medical insurance and the basic medical insurance funds paid by the hospital for the treatment of patients (The basic medical insurance funds paid by the hospital for the treatment of patients, which means that after the end of the patient's diagnosis and treatment, the patient's own medical expenses should be directly settled, and the part of the basic medical insurance fund compensation payment is advanced by the hospital for the patient. The hospital will settle according to the agreed standard and time according to the medical service agreement signed with the basic medical insurance management department). The hospital is prone to losses. For these reasons, in the implementation of the reform of the payment by the single disease, the hospital is likely to cause the standard of patients to be treated or the variants, and the payment by the DRGs (Diagnosis Related Group System) in groups requires scientific and strict disease diagnosis and clinical treatment path. The result of the actual implementation of the reform of the basic medical insurance payment method is not very satisfactory. It is difficult to guide large hospitals to use disease specialists and specialized diseases as development centers to implement the oblique allocation of medical resources.

\section{The Path Choice of the Reform of Basic Medical Insurance Payment Methods}

\subsection{General Principles for Implementing Payment Method Reform}

\subsubsection{Conducive to Improving the Ability of Specialized Disease Services in Large Hospitals}

Large hospitals are generally comprehensive medical institutions at or above the city level. These hospitals are the main medical institutions that undertake the diagnosis and treatment of major diseases and difficult diseases and are the key to solving the problem of difficulty and expensive medical treatment for urban and rural residents. It is necessary to speed up the establishment of open and equal negotiation and consultation mechanisms and risk sharing mechanisms between the basic medical insurance management department (or a specific business agency) and designated medical institutions. [8] Give full play to the various types of medical insurance to regulate, guide and supervise the behavior and expenses of medical services, and effectively control medical costs. Gradually regulate medical insurance to the supervision of medical services to the supervision of medical personnel and medical behavior. [9] Through the medical insurance fund, we will effectively promote the reform and development of large hospitals and effectively improve the ability and level of medical services. Through the reform of payment methods for major diseases and key specialist diseases, the cost of medical treatment for the people is more transparent. The masses can get higher security benefits, and reimbursement can be more convenient and timelier. In particular, patients with similar diseases are promptly concentrated in large hospitals with strong rescue capabilities and good service levels. This will play a positive role in strengthening the construction of specialties and the investment of scientific research resources in large general hospitals.

\subsubsection{Conducive to Improving the Efficiency of Fund Use and Ensuring Fairness}

Promoting the reform of medical insurance payment methods in large hospitals can effectively promote the improvement of the efficiency of basic medical insurance funds and the fairness of basic medical insurance. According to statistics, more than $70 \%$ of patients with major illness insurance for urban and rural residents are seeking medical treatment at provincial and municipal medical institutions, and the cost of major illness insurance is over $80 \%$. [10] The reform of payment methods for major diseases and key specialty diseases was selected. On the one hand, it is possible to gradually build a good order for medical treatment and reasonable order of diagnosis and treatment between large hospitals and primary medical institutions. On the other hand, it can better achieve the benign interaction between the basic medical insurance, medical and medical doctors. Only by doing a good job of reforming the payment methods of large hospitals, it is possible to transfer limited medical insurance funds to medical institutions with scarce resources and high quality of service, in order to better reflect the fairness of the use of medical insurance funds. [11]

\subsubsection{Conducive to Improving the Satisfaction of Patients Seeking Medical Treatment and Compensation}

Through the reform of medical insurance payment methods, patients can choose medical institutions with better services and fairer prices to seek medical treatment according to their preferences. [12] As far as we know, an important 
reason for the current contradiction between doctors and patients is the information asymmetry between doctors and patients. Therefore, through the reform of payment methods, the hospital will increase the transparency of information such as the cost of disease diagnosis and treatment, so that patients have certain choices for medical treatment, which is conducive to improving patient satisfaction with medical treatment.

\subsection{Thoughts on the Path of Reforming Medical Insurance Payment Methods}

\subsubsection{The Hospital will Determine the Type of the Diseases and Enhance the Enthusiasm for Reform}

As a provider of medical services, how to actively integrate into the reform of payment methods is the key to the effectiveness of the payment method reform. In the process of reform and implementation of payment methods in the past, hospitals are often passive recipients of policy formulation. The unified reimbursement policy has made the hospital's autonomous appeals not effectively reflected, and the horizontal differentiation between hospitals cannot be reflected. The selected diseases of the hospital refer to large hospitals (especially provincial and municipal large-scale medical institutions) that can independently select the disease-dependent diseases (DRGs) suitable for their units in light of the relevant requirements of urban public hospital reform. This choice is mainly from the perspective of promoting the development of its key disciplines and effectively attracting patients to participate in basic medical insurance. The hospital needs to take the initiative to report to the basic medical insurance management department. The insurance management organizations organize the calculation and verification work according to the data reported by the hospital, and finally confirms the implementation of the disease types and cost standards of each medical institution and announces the implementation to the public.

\subsubsection{Implement the Same Disease Insurance Standards Are Consistent to Improve the Fairness of Medical Insurance}

In order to ensure the fairness of compensation and respect the right of patients to choose medical institutions, the basic medical insurance management department should adhere to the implementation principle of "the same disease insurance standards are consistent." For the same disease type, the single disease type is paid in a fixed amount, and the medical insurance quota compensation standard remains unchanged. Different medical institutions can reasonably determine the patient's self-paying standards according to the level of their service capabilities, and report them to the medical insurance management agency for approval and approval. For those who pay for "by the diseases," etc., the minimum fee limit of the approved medical institution at the same level is used as the standard compensation report, and the excess is borne by the patient.

\subsubsection{Implement Differentiated Self-Payment, Emphasizing Personal Responsibility}

The cost gap caused by "the same disease insurance standards are consistent" should be compensated by differentiated self-payment (Because the patient chooses different hospitals for diagnosis and treatment of diseases, the cost price of the disease will be different, and the medical expenses of the difference will be borne by the patient.). For medical consumption, the price should be marked clearly, and the price of medical services should match the quality of medical services. For the hospital's special departments, specialty specialists can attract patients through market-oriented pricing strategies. For patients, personal responsibility should be emphasized. Individual medical treatment should not be affected by the fund reimbursement policy. Instead, medical service resources should be selected according to the quality and evaluation of the medical service provider. This will not only help the effective input of medical institutions resources but also promote the fairness and rationality of medical resources allocation. This choice path can give play to the hospital's subjective initiative and significantly improve the health care management effectiveness. The hospital can adjust the price of the disease dynamically according to the patient's treatment situation. The settlement price of the insurance management organizations can be relatively independent and not affected by the price of the hospital disease.

\section{Suggestions on Reforming the Payment Method of Basic Medical Insurance}

In order to make the reform of payment methods in large hospitals more operable, and to enable large disease patients to obtain better services in large hospitals, it is recommended to broaden the reform ideas, integrate social resources, and innovate management and service work. It mainly includes the following aspects.

\subsection{Improve the Effectiveness of the Business by Purchasing Commercial Insurance Company Services}

At present, the government has introduced many policies to encourage the basic medical insurance management department to utilize the market-oriented mechanism in the public service field fully. For example, by purchasing services from commercial insurance companies, it actively explores and promotes qualified commercial insurance institutions to carry out various types of pension and medical insurance. [9] Utilize the professional knowledge of commercial health insurance companies to play the role of their third-party buyers, help alleviate the asymmetry of doctors and patients and contradictions between doctors and patients, and improve the efficiency of social management. [13] Taking the cooperation between China Life Insurance Jiangsu Branch and China Jiangsu Province Medical Insurance Management Center as an example, the country 
took the lead in piloting cooperation with medical insurance provincial-level connected hospitals to carry out resident representative services. China Life Insurance Co., Ltd. mainly cooperates with large urban hospitals to carry out designated services. For example, in Jiangsu Province, services are carried out in large hospitals where people often see a doctor (Mainly refers to participating in basic medical insurance). China Life's designated service will actively assist seriously ill patients to transfer to other provinces for medical treatment and provide full-time assistance services for medical treatment. At present, China Life has been serving in more than 20 provincial and municipal large hospitals such as Jiangsu Provincial People's Hospital, China Jiangsu Provincial Hospital of Traditional Chinese Medicine and Jiangsu Provincial Cancer Hospital of China. If the insured people go to provincial and municipal hospitals to treat large-scale diseases, they can enjoy the "face-to-face" intimate service of the resident representatives, mainly providing referral services, pre-hospital docking, policy consultation, diagnosis and treatment communication, assistance in discharge reimbursement and follow-up after discharge. This approach has established a professional communication and service bridge between the government, hospitals, and patients, which has been affirmed and welcomed by all parties. [14] Under the cooperation mode between China Life Insurance and China's Jiangsu Province Medical Insurance Fund Management Center, provincial and municipal key large hospitals will dispatch full-time service personnel to strengthen guidance and publicity when patients are transferred from various places. Insured people can choose to go to a large hospital with good service, high quality and reasonable cost through this professional service.

\subsection{Bridging the Funding Gap for Basic Health Insurance Through Innovative Commercial Insurance Products}

Germany is the first country in the world to implement a social health insurance model. In recent years, through reforms, commercial insurance companies can operate diversified commercial insurance business based on the government's statutory medical insurance. In Germany, when an individual has an annual income of more than 50,850 euros (2012), his or her individual can choose between legal and commercial insurance. [15] For China, the basic principle of the establishment of the basic medical insurance system is to "guarantee basic and wide coverage." In the case of insufficient compensation for basic medical insurance, including medical expenses for personal self-paying, the state should actively promote the development of commercial health insurance, encourage commercial insurance companies to innovate commercial health insurance products, and meet the multi-level medical security needs of the majority of insured people. [16]

\section{Conclusion}

In order to protect the safety of basic medical insurance funds, efforts are made to improve the efficiency of the use of basic medical insurance funds. At the same time, in order to reasonably guide patients with major diseases to the large hospital for diagnosis and treatment, and to effectively alleviate the cost of expensive hospitals. The current work has become very urgent, that is, to promote the reform of basic medical insurance payment methods in large hospitals. Based on past experience, the government's goal in promoting the reform of basic medical insurance payment methods is not consistent with the goal of large hospitals that they want to develop themselves. Even to a certain extent, it restricts the development of large hospitals, and the actual effect of payment method reform is poor. In order to solve this problem, this article focuses on combing and analyzing China's current policy of reforming the basic medical insurance payment method, and its main features. At the same time, the path selection of the reform of basic medical insurance payment methods was discussed and analyzed in depth.

It is recommended to choose the reform path of basic medical insurance payment methods from three aspects. First, it is conducive to improving the ability of specialized disease services in large hospitals. Second, it is conducive to improving the efficiency of fund use and ensuring fairness. Finally, it is conducive to improving the satisfaction of patients seeking medical treatment and compensation. It is suggested that the Basic Medical Insurance Management Department can implement the "Differentiated Self-Payment" reform idea in accordance with the "The Same Disease Insurance Standards are Consistent" and the individual. Enhance the enthusiasm for reform through the way that the hospital can determine the type of diseases by themselves. Improve the fairness of the guarantee through the same disease insurance standards are consistent. Emphasize personal responsibility by differentiated self-payment. In the end, we will be able to explore new ways to reform the basic medical insurance payment method and implement it in large hospitals. At the same time, it is recommended that the government can purchase the relevant services of commercial insurance companies and make full use of the professional management capabilities of commercial insurance companies. In order to improve the performance of basic medical insurance. This can also play the role of the third party of commercial insurance companies, effectively alleviating the problem of the hospital and patient medical information asymmetry and the contradiction between doctors and patients.

\section{Acknowledgements}

Knowledge is a vast ocean; I am only one of the flatboats. First of all, I am very grateful to my father for leading me into the ocean of knowledge and making me more interested in the management profession. Let me have a glimpse of the vastness of this ocean. Secondly, Thanks to my professor, who greatly improved my understanding of academic writing and taught me a lot of specific research skills. Thanks to the classmates who have given me care and support in life, 
because of you, university life is colorful. Thanks again to my parents, which gives me the opportunity to go abroad, enjoy a more advanced lifestyle, given me a rational look at the world, given me a delicate sense.

\section{References}

[1] Z. "Opinions of the Central Committee of the Communist Party of China and the State Council on Deepening the Reform of the Medical and Health System," China Government Network, 2009, pp. 1-8.

[2] R. Qinyue Meng, Hongwei Yang, Wen Chen, Qiang Sun, Xiaoyun Liu, "China's health system in transition," World Health Organization Asia Pacific Health System and Policy Watch, 2016 (7), pp. 15.

[3] H. Hang Chen, "Medical Reform of The Supply Side_-The study of cooperation mode choice about hierarchical diagnosis and treatment," Beijing: Chemical Industry Press, 2017, pp. $1-10$.

[4] Z. "Several opinions of the State Council's Leading Group on Deepening the Reform of the Medical and Health System on Further Promoting the Experience of Deepening the Reform of the Medical and Health System," China Government Network, 2016, pp. 1-4.

[5] J. Xiaolin He, Ping Jiang, Minjie Zhu, Lei Xu, Wei Lu, "The government collaboration and medical insurance to boost: 'The Four medical linkage' under the protection of family doctor service," Chinese Journal of Health Policy, 2017 (10).

[6] J. Fulin Chu, "Establishing a value-based medical insurance payment system" China Health Insurance, 2017 (11).

[7] Z. "Guiding Opinions of the General Office of the State Council on Further Deepening the Reform of Basic Medical
Insurance Payment Methods," China Government Network, 2017, pp. 1-5.

[8] J. Jingyu Yang, Wu Yan, "The reform of medical insurance payment system is the key to play the basic role of medical insurance," China medical insurance, 2016 (12).

[9] Z. "Guiding Opinions of the General Office of the State Council on the Pilot Reform of Urban Public Hospitals," China Government Network, 2015, pp. 1-4.

[10] J. Debin Xu, Junhua Hua and Weijie Li, "China Life Insurance undertakes the experience and thinking of the major illness insurance for urban and rural residents," Jiangsu Insurance, 2017 (10), pp. 27-30.

[11] J. "Fuling Zhu. Establishing a value-oriented medical insurance payment system," China Medical Insurance, 2017 (11), pp. 34-35.

[12] J. Ting Pan, Wei Du, "The Construction Practice of Wisdom Unified Settlement \& Payment Platform Mode in Wuhai," China Digital Medicine, 2017, 12 (9), pp. 21-23.

[13] Z. "Several opinions of the State Council on accelerating the development of modern insurance service industry," China Government Network, 2014, pp. 1-6.

[14] J. Debin Xu, Aihua Jiang and Yindong Wen, "Commercial insurance company undertakes the risk identification, measurement and control research of urban and rural residents' major illness insurance business," Jiangsu Insurance, 2018 (2), pp. 31-38.

[15] M. Thomson Sarah, Bussen Reinhard, Crivelli Luca, Wynand van de Vend and Carine Van de Voorde, "Statutory health insurance competition in Europe: A four-country comparison," Health Policy, 2013 (109), pp. 209-225.

[16] R. "China commercial health insurance research," China development research foundation, 2017, pp. 14. 